Nervenarzt 2019 $90: 306$

https://doi.org/10.1007/s00115-019-0687-5

Online publiziert: 26. Februar 2019

(c) Springer Medizin Verlag GmbH, ein Teil von Springer Nature 2019

\section{Kommentar}

Zu den Leserbriefen von H. Helmchen (2019) Die kritische Beschäftigung mit alternativen Methoden ist sinnvoll. Nervenarzt https://doi. org/10.1007/s00115-019-0683-9

J. Noth (2019) Verwischte Grenze zwischen evidenzbasierter Medizin und therapeutischen Verfahren. Nervenarzt https://doi.org/10.1007/s00115-019-0684-8 B. Voss (2019) Königin Zeitgeist - Hurra wir knicken ein. Nervenarzt https://doi.org/10. 1007/s00115-019-0685-7

Volker Arolt ${ }^{1}$ Michael Bauer ${ }^{2} \cdot$ Hans-Peter Kapfhammer ${ }^{3}$. Wolfgang Maier ${ }^{4}$. Frank Schneider ${ }^{5}$

${ }^{1}$ Klinik für Psychiatrie und Psychotherapie, Universitätsklinikum Münster, Münster, Deutschland

${ }^{2}$ Klinik und Poliklinik für Psychiatrie und Psychotherapie, Universitätsklinikum Carl Gustav Carus, Technische Universität Dresden, Dresden, Deutschland

${ }^{3}$ Klinik für Psychiatrie und Psychotherapeutische Medizin, Medizinische Universität Graz, Graz, Österreich ${ }^{4}$ Klinik und Poliklinik für Psychiatrie und Psychotherapie, Universitätsklinikum Bonn, Bonn, Deutschland

${ }^{5}$ Universitätsklinikum Düsseldorf, Düsseldorf, Deutschland

\title{
Kommentar zu den Leserbriefen des Leitthemenheftes „Komplementär- und Alternativtherapien für die Psychiatrie“
}

Zum Leitthema der Septemberausgabe 2018 „Komplementär- und Alternativtherapien für die Psychiatrie" haben uns die nachfolgenden Leserbriefe erreicht, die wir hier gerne abdrucken. Da zwei der Briefe sich kritisch äußern, haben wir den verantwortlichen Gastherausgeber gebeten hierzu Stellung zu nehmen.

Als Herausgeber einer wissenschaftlichen Zeitschrift sind wir grundsätzlich den Prinzipien der evidenzbasierten Medizin, wie sie auch in den S2- und S3Leitlinien der medizinischen Fachgesellschaften in Deutschland zur Anwendung kommen, verpflichtet. Das gilt insbesondere für Therapieverfahren. Es gibt aber auch Behandlungsmethoden, die ohne Evidenzbasierung den Weg in die therapeutische Praxis gefunden haben und von Patienten nachgefragt werden. Es ist auch die Aufgabe einer wissenschaftlichen Zeitschrift in diesem ungesicherten Behandlungsfeld Orientierung zu schaffen.

Die psychiatrischen Herausgeber der Zeitschrift Der Nervenarzt

\section{Korrespondenzadresse}

\section{Prof. Dr. Wolfgang Maier}

Klinik und Poliklinik für Psychiatrie und Psychotherapie, Universitätsklinikum Bonn Sigmund-Freud-Straße 25, 53105 Bonn, Deutschland

Wolfgang.maier@ukbonn.de

Interessenkonflikt. V. Arolt, M. Bauer, H.-P. Kapfhammer, W. Maier und F. Schneider geben an, dass kein Interessenkonflikt besteht. 